\title{
Glucose Effectiveness from Short Insulin-Modified IVGTT and Its Application to the Study of Women with Previous Gestational Diabetes Mellitus
}

\author{
Micaela Morettini ${ }^{1}$, Carlo Castriota ${ }^{1}$, Christian Göbl ${ }^{2}$, Alexandra Kautzky-Willer ${ }^{3}$, Giovanni Pacini ${ }^{4}$, Laura Burattini ${ }^{1}$, Andrea Tura ${ }^{4}$ \\ ${ }^{1}$ Department of Information Engineering, Università Politecnica delle Marche, Ancona, Italy, \\ ${ }^{2}$ Division of Obstetrics and Feto-Maternal Medicine, Department of Obstetrics and Gynecology, Medical University of Vienna, Vienna, \\ ${ }^{3}$ Division of Endocrinology and Metabolism, Department of Internal Medicine III, Medical University of Vienna, Vienna, Austria, \\ ${ }^{4}$ Metabolic Unit, CNR Institute of Neuroscience, Padova, Italy
}

Background: This study aimed to design a simple surrogate marker (i.e., predictor) of the minimal model glucose effectiveness $\left(\mathrm{S}_{\mathrm{G}}\right)$, namely calculated $\mathrm{S}_{\mathrm{G}}\left(\mathrm{CS}_{\mathrm{G}}\right)$, from a short insulin-modified intravenous glucose tolerance test (IM-IVGTT), and then to apply it to study women with previous gestational diabetes mellitus (pGDM).

Methods: $\mathrm{CS}_{\mathrm{G}}$ was designed using the stepwise model selection approach on a population of subjects $(n=181)$ ranging from normal tolerance to type 2 diabetes mellitus (T2DM). $\mathrm{CS}_{\mathrm{G}}$ was then tested on a population of women with pGDM $(n=57)$. Each subject underwent a 3-hour IM-IVGTT; women with PGDM were observed early postpartum and after a follow-up period of up to 7 years and classified as progressors (PROG) or non-progressors (NONPROG) to T2DM. The minimal model analysis provided a reference $\mathrm{S}_{\mathrm{G}}$.

Results: $\mathrm{CS}_{\mathrm{G}}$ was described as $\mathrm{CS}_{\mathrm{G}}=1.06 \times 10^{-2}+5.71 \times 10^{-2} \times \mathrm{K}_{\mathrm{G}} / \mathrm{G}_{\text {peak }}, \mathrm{K}_{\mathrm{G}}$ being the mean slope (absolute value) of $\log _{\mathrm{e}}$ glucose in 10-25- and 25-50-minute intervals, and $\mathrm{G}_{\text {peak }}$ being the maximum of the glucose curve. Good agreement between $\mathrm{CS}_{\mathrm{G}}$ and $\mathrm{S}_{\mathrm{G}}$ in the general population and in the pGDM group, both at baseline and follow-up (even in PROG and NONPROG subgroups), was shown by the Bland-Altman plots ( $<5 \%$ observations outside limits of agreement), and by the test for equivalence (equivalence margin not higher than one standard deviation). At baseline, the PROG subgroup showed significantly lower $\mathrm{S}_{\mathrm{G}}$ and $\mathrm{CS}_{\mathrm{G}}$ values compared to the NONPROG subgroup $(P<0.03)$.

Conclusion: $\mathrm{CS}_{\mathrm{G}}$ is a valid $\mathrm{S}_{\mathrm{G}}$ predictor. In the $\mathrm{pGDM}$ group, glucose effectiveness appeared to be impaired in women progressing to T2DM.

Keywords: Diabetes, gestational; Diabetes mellitus, type 2; Glucose metabolism disorders; Glucose tolerance test; Models, theoretical

\section{INTRODUCTION}

The term 'glucose effectiveness' has been used to indicate and quantify the processes that occur to normalize the glucose concentration during hyperglycemia, independently from any change in insulin concentration [1]. In fact, glucose per se has the ability to stimulate its own uptake and suppress its own production even at basal insulin concentrations [1], contributing to about $60 \%$ of all glucose disposal in normal human subjects and animals [1-3]. The role of glucose effectiveness in predicting possible future derangements of glucose tolerance has gained increasing interest in the last years and the available evidence has been recently reviewed by Alford et al. [4], concluding that a lower glucose effectiveness over time is a critical
Corresponding author: Andrea Tura (D) https://orcid.org/0000-0003-3466-5900 Metabolic Unit, CNR Institute of Neuroscience, Corso Stati Uniti 4, Padova 35127, Italy E-mail: andrea.tura@cnr.it

Received: Jan. 22, 2019; Accepted: May 24, 2019
This is an Open Access article distributed under the terms of the Creative Commons Attribution Non-Commercial License (https://creativecommons.org/licenses/by-nc/4.0/) which permits unrestricted non-commercial use, distribution, and reproduction in any medium, provided the original work is properly cited. 
determinant of future glucose intolerance and type 2 diabetes mellitus (T2DM) in subjects at increased risk. Recent evidence has also shown that overweight and obese subjects, who are at recognized increased risk of developing T2DM, exhibit lower glucose effectiveness [5]. On this basis, specific therapies aimed at enhancing glucose effectiveness in at-risk or intolerant subjects may play an important role in preventing T2DM [4]. Such treatments may include sodium glucose cotransporter 2 (SGLT2) inhibitors, recently proposed as antidiabetic pharmacologic agents, which exert their function by acting directly on non-insulin-mediated processes [6]. Thus, quantification of glucose effectiveness should be performed to have a complete description of the glucose tolerance status.

The history of gestational diabetes mellitus (GDM) is one of the most representative conditions leading to the increased risk for developing glucose intolerance and eventually T2DM [7], thus suggesting regular follow-ups for early detection of possible deterioration of glucose tolerance. Women with previous GDM (pGDM), among several metabolic abnormalities, may also exhibit lower glucose effectiveness [8]. However, to the best of our knowledge, no study has focused on the analysis of glucose effectiveness in this population; specifically, possible alterations in glucose effectiveness after a follow-up period have never been explored in pGDM.

Originally, two tests were employed to assess glucose effectiveness: the hyperglycemic glucose clamp and the intravenous glucose tolerance test (IVGTT) with minimal model interpretation $[3,9]$. The estimation of glucose effectiveness provided by the minimal model, known as the $\mathrm{S}_{\mathrm{G}}$ index, has been proven to be reliable and equivalent to the one provided by the clamp technique [10]. Although less experimentally demanding compared to the clamp technique, the minimal model $\mathrm{S}_{\mathrm{G}}$ assessment requires a 3-hour IVGTT, specific skills, and dedicated computer programs for model parameter estimation. To overcome these limitations, a simple index, calculated $\mathrm{S}_{\mathrm{G}}\left(\mathrm{CS}_{\mathrm{G}}\right)$, has been recently proposed, allowing the reliable assessment of $\mathrm{S}_{\mathrm{G}}$ without using complex mathematical models and resorting to a shorter-only 1-hour-IVGTT [11].

It has been shown that increasing the dynamics of the IVGTT may yield more accurate evaluation of the metabolic parameters. To this aim, an infusion of insulin can be added to the traditional IVGTT, a test termed the insulin-modified IVGTT (IM-IVGTT). This test is particularly recommended in subjects at increased risk of developing T2DM, such as women with PGDM, where the IM-IVGTT has been used to accurately assess possible defects in insulin sensitivity [12]. A simple index has already been proposed to assess insulin sensitivity [13] from the IM-IVGTT, but this has not been conducted for glucose effectiveness. Thus, the aims of this study were: (1) to derive-from a short IM-IVGTT-a simple surrogate marker (i.e., a predictor) of the $\mathrm{S}_{\mathrm{G}}$; (2) to test the reliability of the derived surrogate marker in a population of women with pGDM; and (3) to analyze possible alterations in glucose effectiveness in this population, at baseline and after a follow-up period.

\section{METHODS}

\section{Participants}

This study has been granted the exemption from approval by Marche Regional Ethics Committee, being a retrospective analysis of datasets collected and published from previous studies [7,14-20]. Informed consent is not applicable due to a retrospective study. The original studies [7,14-20] were performed in agreement with the Declaration of Helsinki, were approved by the respective local ethics committees and all participants provided their informed consent.

Participants included subjects with different clinical characteristics, determining different glucose tolerance statuses: (1) nondiabetic subjects (ND, $n=108$ ), including subjects having normal glucose regulation and subjects affected by conditions causing a possible deterioration in glucose regulation (e.g., obesity) and (2) subjects with T2DM ( $n=73)$. These subjects represented the training dataset. Women with pGDM $(n=57)$, already analyzed in a previous study [20], were also considered. All women were analyzed early postpartum (4 to 6 months after delivery) and then re-examined over a period of up to 7 years. All women were nondiabetic at the time of the first analysis (early postpartum). None of the women was treated with antidiabetic agents before the possible onset of T2DM.

All participants underwent a 3-hour IM-IVGTT, with an intravenous insulin infusion $(0.03$ to $0.05 \mathrm{U} / \mathrm{kg})$ at 20 minutes after the glucose infusion $(0.3 \mathrm{~g} / \mathrm{kg})$. Blood samples were collected during the 3-hour period, with frequent sampling during the first 30 minutes (typically at time $0,3,4,5,6,8,10,14$, $19,22,27,30,35,40,50,70,100,140,180$ minutes), for the measurement of glucose and insulin concentrations. In some cases, blood samples were collected with a slightly different sampling protocol, resulting in the replacement of some of the 
reported samples with additional samples (e.g., at time 2, 12, $15,20,25,60,80$ minutes). The women in the pGDM group underwent an IM-IVGTT both at baseline and at the last follow-up visit. Since some women developed T2DM during the study, they were divided into two groups: those progressing to T2DM (progressors [PROG]) and those remaining nondiabetic (nonprogressors [NONPROG]). For the PROG subgroup, the last follow-up visit was at the onset of the disease (shortly thereafter), whereas for the NONPROG subgroup, the last follow-up visit occurred at least 5 years after the first visit (up to a maximum of 7 years). The main characteristics of the participants are reported in Table 1.

\section{Calculations and statistical analysis}

In all participants, $\mathrm{S}_{\mathrm{G}}(1 / \mathrm{min})$ was assessed by minimal model analysis of IM-IVGTT data [14]. The $\mathrm{CS}_{\mathrm{G}}(1 / \mathrm{min})$ index was designed and validated on the indicated subjects with different clinical characteristics (ND and T2DM groups). The $\mathrm{CS}_{\mathrm{G}}$ design was performed through a stepwise model selection approach by optimizing the Akaike's information criterion, using the minimal model $\mathrm{S}_{\mathrm{G}}(1 / \mathrm{min})$ as a dependent variable. As an independent variable, the rate of glucose disappearance $K_{G}(1 / \mathrm{min})$ - normalized to the maximum of the glucose curve during the test $\mathrm{G}_{\text {peak }}(\mathrm{mmol} / \mathrm{L})$-was assumed as the key factor for the prediction of $\mathrm{S}_{\mathrm{G}}$, as previously demonstrated for regular IVGTT [11]. $K_{\mathrm{G}}$ was computed as the mean value between $\mathrm{K}_{\mathrm{G} 1}$ and $\mathrm{K}_{\mathrm{G} 2}$, i.e., the slope (absolute value) of $\log _{e}$ glucose multiplied by 100 in the 10-25- and 25-50-minute intervals, respectively, as already performed in a previous study assessing insulin sensitivity from the short IM-IVGTT [13]. The 25-minute time-point was selected since the insulin infusion took place at 20 minutes and lasted for approximately 1 minute; thus, 5 minutes could be a reasonable delay for exogenous insulin to act on glucose disappearance. Other independent variables included in the stepwise model selection were age, sex, body mass index (BMI), basal glucose, and insulin concentrations.

Similarly to other studies $[11,21]$, the $\mathrm{CS}_{\mathrm{G}}$ formulation obtained from the stepwise model selection was validated using the "jackknife" technique, which allowed for the estimation of index prospective accuracy when applied to an independent set of subjects [22]. To this purpose, one subject was removed from the dataset, and model coefficients were computed on the remaining subjects. Based on such model coefficients, a predicted $\mathrm{CS}_{\mathrm{G}}$ value was computed for the removed subject, and this was repeated for every subject. Furthermore, the derived $\mathrm{CS}_{\mathrm{G}}$ formula was then tested for the assessment of glucose effectiveness in the pGDM group (and in its related PROG and NONPROG subgroups), both at baseline and at follow-up.

To evaluate the degree of agreement between $\mathrm{CS}_{\mathrm{G}}$ and $\mathrm{S}_{\mathrm{G}}$, Bland-Altman plots were computed. Finally, a Two One-Sided Test for equivalence (TOST) [11,23], was performed to test the minimum equivalence margin (epsilon parameter) showing equivalence between $S_{\mathrm{G}}$ and $\mathrm{CS}_{\mathrm{G}}$.

After stratifying the pGDM group into the PROG and NONPROG subgroups, an unpaired Student $t$-test was performed to assess differences in $\mathrm{S}_{\mathrm{G}}$ and $\mathrm{CS}_{\mathrm{G}}$ at baseline and at follow-up.

Following the Kolmogorov-Smirnov test for normality, in the case of skewed distributions, tests were applied to the logtransformed values. Values are reported as mean \pm standard er-

Table 1. Characteristics of the participants

\begin{tabular}{|c|c|c|c|c|c|}
\hline Characteristic & Sex, male/female & Age, yr & BMI, $\mathrm{kg} / \mathrm{m}^{2}$ & Basal glucose, mmol/L & Basal insulin, pmol/L \\
\hline $\mathrm{ND}$ & $18 / 90$ & $33.1 \pm 1.0$ & $28.2 \pm 0.7$ & $5.0 \pm 0.1$ & $67.5 \pm 4.5$ \\
\hline T2DM & $49 / 24$ & $48.8 \pm 1.9$ & $30.0 \pm 0.6$ & $9.1 \pm 0.3$ & $86.4 \pm 7.3$ \\
\hline \multicolumn{6}{|l|}{ pGDM } \\
\hline \multicolumn{6}{|l|}{ NONPROG } \\
\hline Baseline & $0 / 43$ & $33.9 \pm 0.6$ & $25.3 \pm 0.6$ & $4.7 \pm 0.1$ & $50.5 \pm 5.0$ \\
\hline Follow-up & & $38.9 \pm 0.6$ & $25.2 \pm 0.6$ & $4.9 \pm 0.1$ & $58.5 \pm 4.8$ \\
\hline \multicolumn{6}{|l|}{ PROG } \\
\hline Baseline & $0 / 14$ & $36.6 \pm 1.3$ & $32.5 \pm 1.8$ & $5.7 \pm 0.5$ & $92.1 \pm 24.6$ \\
\hline Follow-up & & $41.3 \pm 1.5$ & $34.2 \pm 2.1$ & $6.9 \pm 0.4$ & $107.7 \pm 17.1$ \\
\hline
\end{tabular}

Values are presented as mean \pm standard error of the mean.

BMI, body mass index; ND, nondiabetic subjects; T2DM, type 2 diabetes mellitus; pGDM, previous gestational diabetes mellitus; NONPROG, women not progressing to type 2 diabetes mellitus at follow-up; PROG, women progressing to type 2 diabetes mellitus. 
ror of the mean, unless otherwise specified. Statistical analysis was performed in R V3.4.3 (The R Foundation for Statistical Computing Platform, Vienna, Austria). The two-sided significance level was set at 5\% $(P<0.05)$.

\section{RESULTS}

The stepwise model selection showed that, among all the tested explanatory variables, $\mathrm{K}_{\mathrm{G}} / \mathrm{G}_{\text {peak }}$ was the only variable that had a significant role in predicting the minimal model $\mathrm{S}_{\mathrm{G}}$. Thus, the $\mathrm{CS}_{\mathrm{G}}$ formulation was:

$$
C S_{G}=1.06 \times 10^{-2}+5.71 \times 10^{-2} \frac{\mathrm{K}_{\mathrm{G}}}{\mathrm{G}_{\text {peak }}}
$$

The related Bland-Altman plots (in the training dataset and in the jackknife-obtained validation dataset) (Fig. 1) showed a substantial agreement between $\mathrm{CS}_{\mathrm{G}}$ and $\mathrm{S}_{\mathrm{G}}$, with less than $5 \%$ of the data falling outside the limits of agreement and no fixed bias affecting $\mathrm{CS}_{\mathrm{G}}$ estimation (mean of $\mathrm{S}_{\mathrm{G}}-\mathrm{CS}_{\mathrm{G}}$ not significantly different from zero). Furthermore, according to the TOST procedure, $\mathrm{CS}_{\mathrm{G}}$ and $\mathrm{S}_{\mathrm{G}}$ were shown to be equivalent both in the training and validation dataset, with small equivalence margins: epsilon was in fact equal to 0.1 standard deviation (SD) of $\mathrm{S}_{\mathrm{G}}$ in both cases.

With regard to the pGDM group, the mean follow-up duration in the NONPROG subgroup was $5.2 \pm 0.1$ years. In the PROG subgroup, the follow-up was interrupted at the onset of T2DM: the mean follow-up duration was $4.6 \pm 0.4$ years. Notably, in our dataset, almost $80 \%$ (11 out of 14) of those women
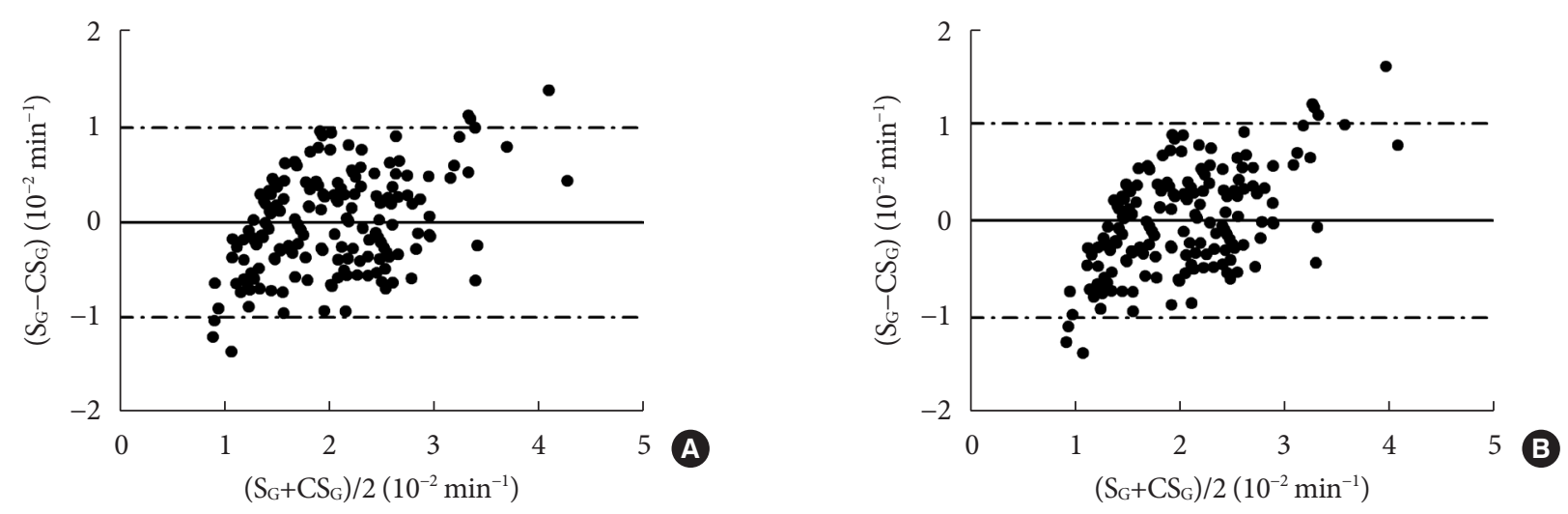

Fig. 1. Bland-Altman plot for the training dataset (A) and the jackknife-based validation dataset (B) in a population of subjects with different clinical characteristics (nondiabetic [ND] and type 2 diabetes mellitus [T2DM] groups). The continuous line represents the mean of the difference between minimal model glucose effectiveness $\left(\mathrm{S}_{\mathrm{G}}\right)$ and calculated $\mathrm{S}_{\mathrm{G}}\left(\mathrm{CS}_{\mathrm{G}}\right)$; the dash-dotted lines represent the limits of agreement (mean $\pm 1.96 \times$ standard deviation).
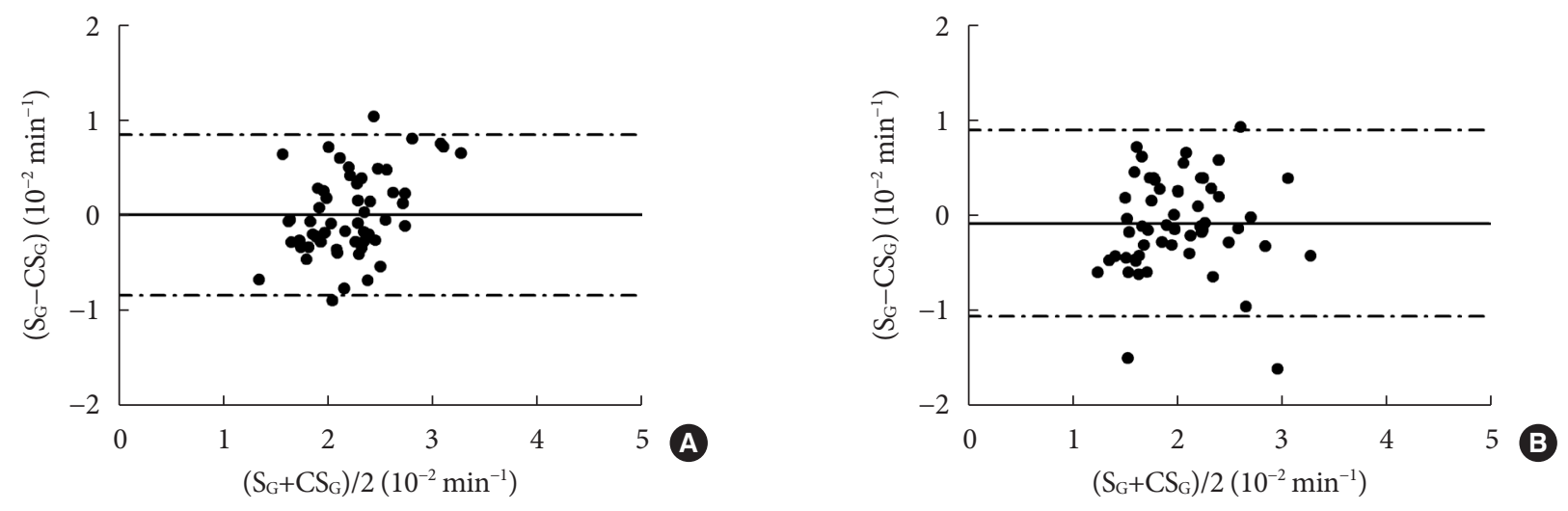

Fig. 2. Bland-Altman plot for the previous gestational diabetes mellitus group (women) at baseline (A) and follow-up (B). The continuous line represents the mean of the difference between minimal model glucose effectiveness $\left(\mathrm{S}_{\mathrm{G}}\right)$ and calculated $\mathrm{S}_{\mathrm{G}}\left(\mathrm{CS}_{\mathrm{G}}\right)$; the dash-dotted lines represent the limits of agreement (mean $\pm 1.96 \times$ standard deviation). 

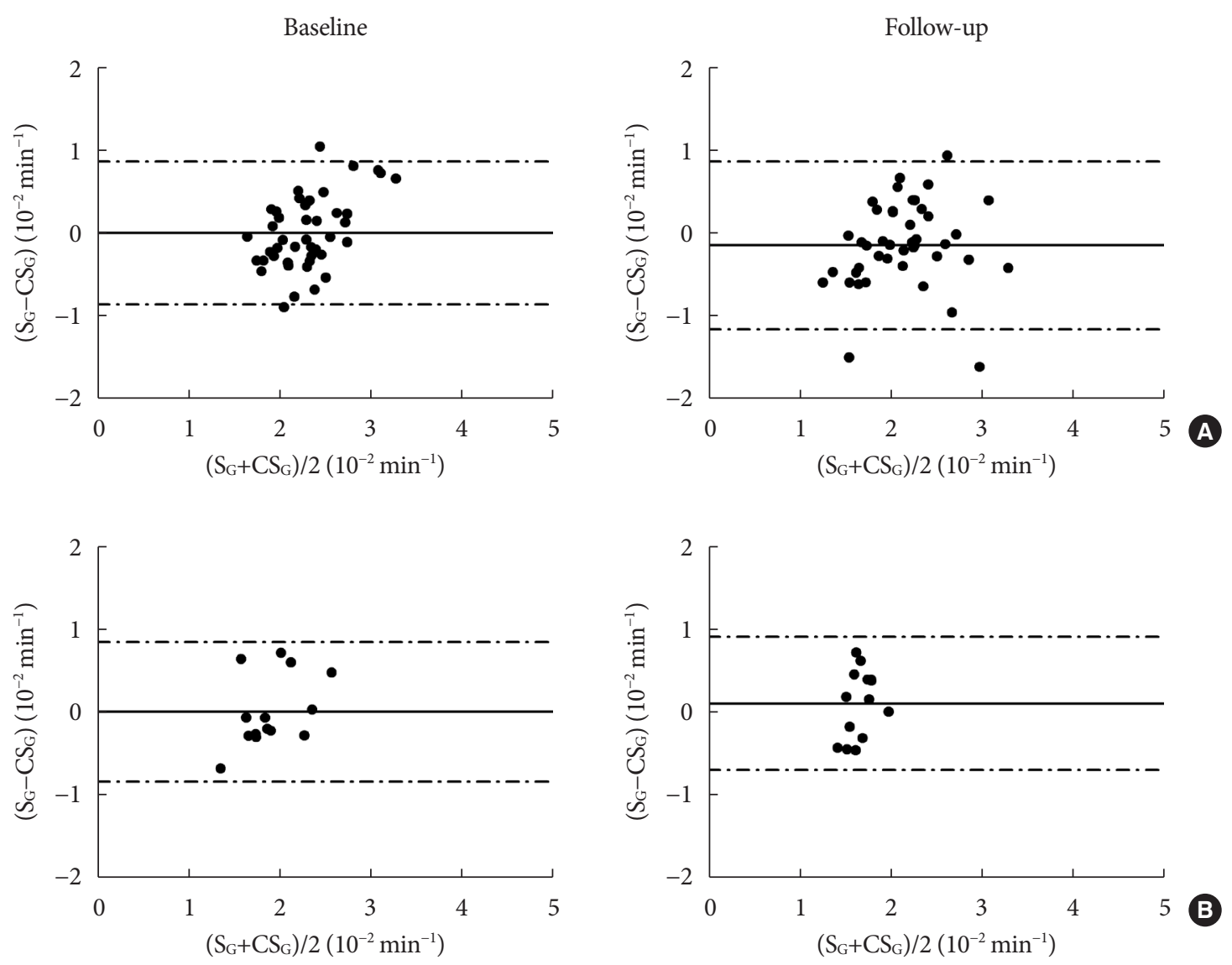

Fig. 3. Bland-Altman plot for the (A) women not progressing to type 2 diabetes mellitus (NONPROG) and (B) women progressing to type 2 diabetes mellitus (PROG) subgroups at baseline and follow-up. The continuous line represents the mean of the difference between minimal model glucose effectiveness $\left(\mathrm{S}_{\mathrm{G}}\right)$ and calculated $\mathrm{S}_{\mathrm{G}}\left(\mathrm{CS}_{\mathrm{G}}\right)$; the dash-dotted lines represent the limits of agreement (mean $\pm 1.96 \times$ standard deviation).

progressing to T2DM developed the disease within 5 years of follow-up.

In the pGDM group and in the related NONPROG and PROG subgroups, Bland-Altman plots at baseline and at follow-up (Figs. 2 and 3) showed substantial agreement between $\mathrm{CS}_{\mathrm{G}}$ and $\mathrm{S}_{\mathrm{G}}$, again with less than $5 \%$ of the data falling outside the limits of agreement, with no fixed bias affecting $\mathrm{CS}_{\mathrm{G}}$ estimation. Also, in the $\mathrm{pGDM}$ group, $\mathrm{CS}_{\mathrm{G}}$ and $\mathrm{S}_{\mathrm{G}}$ were shown to be equivalent according to the TOST procedure, both at baseline and at follow-up, with the equivalence margin epsilon equal to $0.2 \mathrm{SD}$ and $0.3 \mathrm{SD}$, respectively. In the NONPROG and PROG subgroups, at baseline, the epsilon was equal to 0.3 and $0.5 \mathrm{SD}$, respectively; at follow-up, the epsilon was equal to 0.5 and $1 \mathrm{SD}$, respectively.

Glucose effectiveness values assessed by $\mathrm{S}_{\mathrm{G}}$ and $\mathrm{CS}_{\mathrm{G}}$ at baseline and at follow-up in the PROG and NONPROG subgroups are reported in Fig. 4. At baseline, the PROG subgroup showed significantly lower $\mathrm{S}_{\mathrm{G}}$ and $\mathrm{CS}_{\mathrm{G}}$ values compared to the NONPROG subgroup ( $P=0.01$ and $P<0.0001$, respectively). Similar results were found at follow-up $(P=0.03$ and $P<0.0001$, respectively).

\section{DISCUSSION}

The present study proposed and validated a simple index for the assessment of $\mathrm{CS}_{\mathrm{G}}$ that predicts the minimal model $\mathrm{S}_{\mathrm{G}}$, when a simple 1-hour IM-IVGTT is performed. IM-IVGTT may be preferred to the regular IVGTT in subjects with high insulin resistance, to obtain a more reliable estimation of this metabolic parameter [14,24-26]. The introduction of $\mathrm{CS}_{\mathrm{G}}$, together with the previously proposed calculated insulin sensitivity $\left(\mathrm{CS}_{\mathrm{I}}\right)$ [13], overcomes the limitations of minimal model 
Baseline
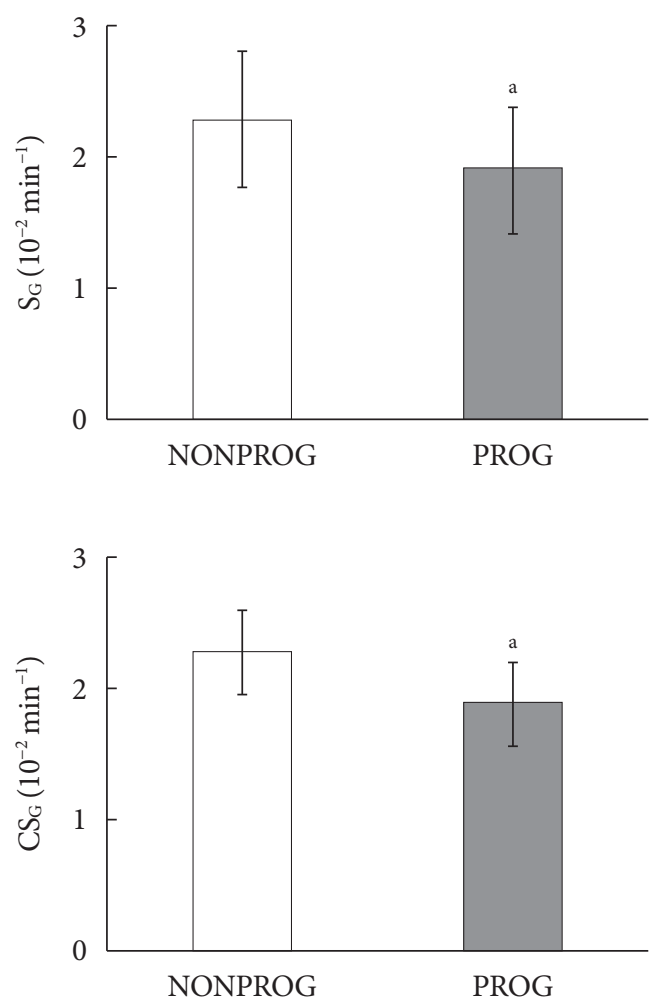

Follow-up
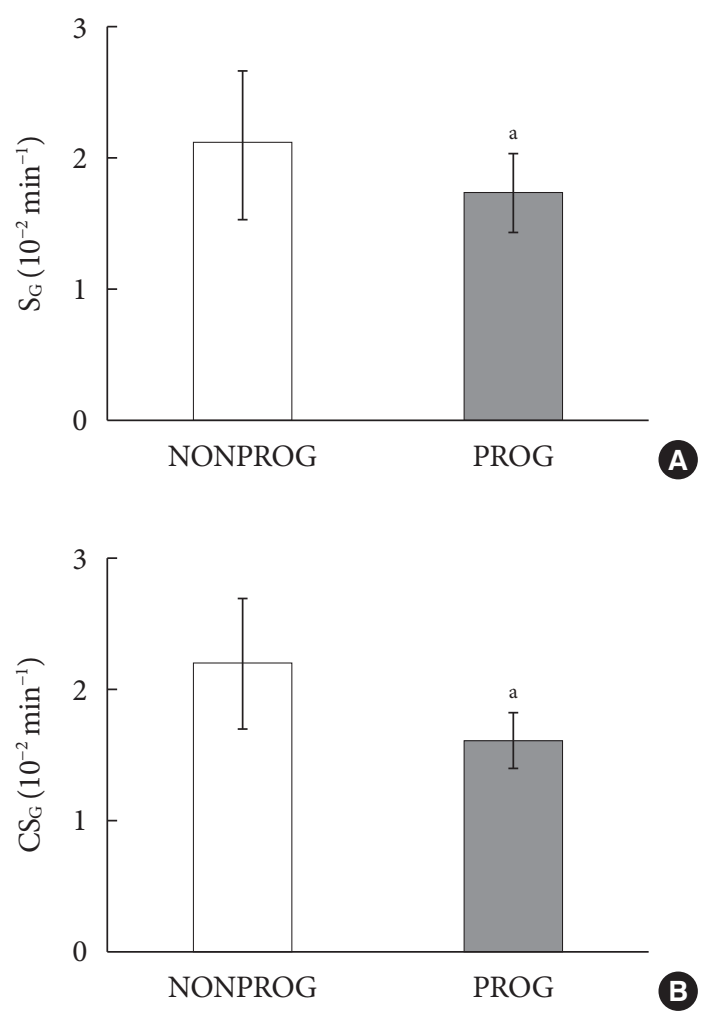

Fig. 4. Glucose effectiveness assessed by $(A)$ minimal model glucose effectiveness $\left(\mathrm{S}_{\mathrm{G}}\right)$ and $(\mathrm{B})$ calculated $\mathrm{S}_{\mathrm{G}}\left(\mathrm{CS}_{\mathrm{G}}\right)$ at baseline and follow-up in the women not progressing to type 2 diabetes mellitus at follow-up (NONPROG) and women progressing to type 2

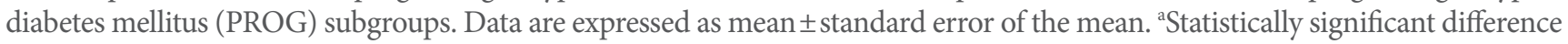
$(P<0.05)$.

methodology, allowing a simple assessment of the two main processes regulating glucose disposal from a short, 1-hour, IMIVGTT. The $\mathrm{CS}_{\mathrm{G}}$ index proposed in this study exhibited excellent performances on the general population ranging from normal glucose tolerance to T2DM, as shown by the BlandAltman plot (Fig. 1) and by the results of the test for equivalence (TOST), showing an equivalence margin parameter much lower than the $\mathrm{SD}$ of $\mathrm{S}_{\mathrm{G}}$.

The $\mathrm{CS}_{\mathrm{G}}$ index was then tested in a population of women with a previous history of GDM, commonly recognized for an increased risk of developing T2DM. This population was analyzed early postpartum and then re-examined up to 7 years later. To the best of our knowledge, this is the first study to analyze, in detail, the glucose effectiveness in women with pGDM, coupled with a specific focus on the possible differences of this metabolic parameter in women progressing to T2DM during a follow-up period, compared to women that did not progress to T2DM. As detected by values at baseline and at follow-up, we found that glucose effectiveness was significantly and chronically reduced in those women progressing to T2DM compared to women remaining nondiabetic (Fig. 4). These findings suggest that in this population of women who progress to T2DM, alterations in glucose effectiveness, together with the already well-known defects in insulin resistance and secretion, could contribute to the development of T2DM. In fact, results obtained in previous studies showed that glucose effectiveness independently predicts conversion to T2DM across ethnic groups, family history of T2DM, and obesity [27]. Notably, a substantial equivalence between $\mathrm{S}_{\mathrm{G}}$ and $\mathrm{CS}_{\mathrm{G}}$ was proven again by the Bland-Altman plots (Figs. 2 and 3 ) and by the TOST analysis (showing equivalence margin always not higher than one SD), both at baseline and at follow-up.

In a previous study, $\mathrm{CS}_{\mathrm{G}}$ was assessed from a regular 1-hour IVGTT [11]. Comparing the "regular" and the "insulin-modified" indices, one can notice that the formulation is essentially the same, i.e., the ratio between the rate of glucose disappear- 
ance, $\mathrm{K}_{\mathrm{G}}$, and the maximum of the glucose curve during the test, $\mathrm{G}_{\text {peak }}$. This confirms the central role assumed by $\mathrm{K}_{\mathrm{G}} / \mathrm{G}_{\text {peak }}$ in the prediction of $\mathrm{S}_{\mathrm{G}}$. Conversely, other regressors (sex, age, BMI, basal glucose, and insulin concentrations), which were proven to be relevant variables in other studies [28], were not able to improve the $S_{\mathrm{G}}$ prediction.

The proposed $\mathrm{CS}_{\mathrm{G}}$ index was designed and validated on a general population, including subjects with glucose tolerance abnormalities, ranging from defective glucose regulation to overt T2DM, but also healthy subjects. Insulin administration during IM-IVGTT can induce transient hypoglycemia and a consequent counterregulatory response in healthy insulinsensitive subjects, thus determining the inaccuracy of minimal model-based parameter estimation [29]. However, it was shown that this inaccuracy pertains only to $S_{\mathrm{I}}$ and not to $S_{\mathrm{G}}$ estimation [29], making inclusion of insulin-sensitive healthy subjects in $\mathrm{CS}_{\mathrm{G}}$ design an advantage rather than a disadvantage.

Since this study represents a post hoc analysis of datasets collected from previous studies, IM-IVGTT data were obtained according to different insulin administration protocols (i.e., 0.03 or $0.05 \mathrm{U} / \mathrm{kg}$ at 20 minutes). The choice to include data derived from different protocols has been justified by the observation that using different insulin doses and protocols during the IM-IVGTT does not affect the minimal model estimation of $\mathrm{S}_{\mathrm{G}}[30]$. Also, $\mathrm{K}_{\mathrm{G}}$ estimation is not affected by the insulin dose [30]. Moreover, the sampling protocol was not exactly the same in all studies. Nonetheless, the obtained results have proven the robustness of the proposed $\mathrm{CS}_{\mathrm{G}}$, which can be computed on the basis of somewhat different IVGTT protocols.

In this study, $\mathrm{K}_{\mathrm{G}}$ was computed as the mean value between $\mathrm{K}_{\mathrm{G} 1}$ and $\mathrm{K}_{\mathrm{G} 2}$, i.e., the rates of glucose disappearance before (10 to 25 minutes) and after ( 25 to 50 minutes) the insulin infusion, as previously carried out for $\mathrm{CS}_{\mathrm{I}}$ [13]. In the pGDM group, we analyzed the effect of replacing $K_{G}$ with $K_{G 1}$ in the equation (as above) for $\mathrm{CS}_{\mathrm{G}}$ assessment. Since samples drawn before the 25-minute are not influenced by insulin infusion, using $\mathrm{K}_{\mathrm{G} 1}$ rather than $\mathrm{K}_{\mathrm{G}}$ would mean resorting to a regular, very short, IVGTT. In that condition, $\mathrm{CS}_{\mathrm{G}}$ estimation was not equivalent to $S_{\mathrm{G}}$ (results not shown), corroborating the hypothesis that IM-IVGTT may be superior to regular IVGTT; thus, it should be preferred when possible.

Important factors differentiating women at highest risk of progression to T2DM among all the PGDM women are family history of diabetes, certain ethnicities, age, early onset of
GDM, hypertension during pregnancy, high BMI, insulin resistance, impaired $\beta$-cell function [31]. However, other possible markers for later risk of T2DM have been hypothesized recently. For instance, an increased circulating fatty acid-binding protein 4 (FABP4) has been shown to differentiate between women progressing to and those not progressing to T2DM after GDM [32]. In fact, circulating FABP4 has pleiotropic roles that include the stimulation of hepatic glucose production [33]. On the other hand, glucose effectiveness is defined as the ability of glucose per se to stimulate its own uptake, but also to suppress its own production [1]. Thus, the lower glucose effectiveness characterizing the $\mathrm{pGDM}$ women progressing to T2DM may reflect, among other factors, increased levels of FABP4, which have shown to be a condition of risk for T2DM progression [32]. However, further studies are required to corroborate this hypothesis.

It is also worth noting that all the subjects included in this study were white Caucasians, and hence, the application of $\mathrm{CS}_{\mathrm{G}}$ to assess glucose effectiveness in other ethnicities (e.g., Asian subjects) has to be tested.

In conclusion, this study developed a simple index for the assessment of glucose effectiveness when an IM-IVGTT is performed. Testing the new index, $\mathrm{CS}_{\mathrm{G}}$, in a population of women with a previous history of GDM confirmed the index reliability in a category of subjects at a particularly high risk for developing T2DM. Since glucose effectiveness appears to be impaired in this population of subjects progressing to T2DM development, its simple and reliable quantification by $\mathrm{CS}_{\mathrm{G}}$ may facilitate a timely assessment of this aspect of glucose metabolism, thus possibly prompting corrective actions that have now become available with pharmacological agents, such as SGLT2 inhibitors, which act independently on insulin.

\section{CONFLICTS OF INTEREST}

No potential conflict of interest relevant to this article was reported.

\section{AUTHOR CONTRIBUTIONS}

Conception or design: M.M., G.P., A.T.

Acquisition, analysis, or interpretation of data: M.M., C.C., C.G., A.K.W., G.P., L.B., A.T.

Drafting the work or revising: M.M., C.C., C.G., A.K.W., G.P., L.B., A.T. 
Final approval of the manuscript: M.M., C.C., C.G., A.K.W., G.P., L.B., A.T.

\section{ORCID}

Micaela Morettini https://orcid.org/0000-0002-8327-8379

Andrea Tura https://orcid.org/0000-0003-3466-5900

\section{ACKNOWLEDGMENTS}

We would like to thank Angelo Avogaro (University of Padova, Italy), Bernhard Ludvik (Wiener Krankenanstaltenverbund, Austria), John J. Nolan (Trinity College Dublin, Ireland), and Donal O'Gorman (Dublin City University, Ireland) for granting the permission to use the data collected in previous studies.

\section{REFERENCES}

1. Pacini G, Thomaseth K, Ahren B. Contribution to glucose tolerance of insulin-independent vs. insulin-dependent mechanisms in mice. Am J Physiol Endocrinol Metab 2001;281: E693-703.

2. Kahn SE, Prigeon RL, McCulloch DK, Boyko EJ, Bergman RN, Schwartz MW, Neifing JL, Ward WK, Beard JC, Palmer JP. The contribution of insulin-dependent and insulin-independent glucose uptake to intravenous glucose tolerance in healthy human subjects. Diabetes 1994;43:587-92.

3. Ader M, Ni TC, Bergman RN. Glucose effectiveness assessed under dynamic and steady state conditions. Comparability of uptake versus production components. J Clin Invest 1997;99: 1187-99.

4. Alford FP, Henriksen JE, Rantzau C, Beck-Nielsen H. Glucose effectiveness is a critical pathogenic factor leading to glucose intolerance and type 2 diabetes: an ignored hypothesis. Diabetes Metab Res Rev 2018;34:e2989.

5. Morettini M, Di Nardo F, Ingrillini L, Fioretti S, Gobl C, Kautzky-Willer A, Tura A, Pacini G, Burattini L. Glucose effectiveness and its components in relation to body mass index. Eur J Clin Invest 2019;49:e13099.

6. Seufert J. SGLT2 inhibitors: an insulin-independent therapeutic approach for treatment of type 2 diabetes: focus on canagliflozin. Diabetes Metab Syndr Obes 2015;8:543-54.

7. Winzer C, Wagner O, Festa A, Schneider B, Roden M, Bancher-Todesca D, Pacini G, Funahashi T, Kautzky-Willer A. Plasma adiponectin, insulin sensitivity, and subclinical inflamma- tion in women with prior gestational diabetes mellitus. Diabetes Care 2004;27:1721-7.

8. Kautzky-Willer A, Krssak M, Winzer C, Pacini G, Tura A, Farhan S, Wagner O, Brabant G, Horn R, Stingl H, Schneider B, Waldhausl W, Roden M. Increased intramyocellular lipid concentration identifies impaired glucose metabolism in women with previous gestational diabetes. Diabetes 2003;52:244-51.

9. Pacini G, Bergman RN. MINMOD: a computer program to calculate insulin sensitivity and pancreatic responsivity from the frequently sampled intravenous glucose tolerance test. Comput Methods Programs Biomed 1986;23:113-22.

10. Henriksen JE, Alford F, Ward G, Thye-Ronn P, Levin K, Hother-Nielsen O, Rantzau C, Boston R, Beck-Nielsen H. Glucose effectiveness and insulin sensitivity measurements derived from the non-insulin-assisted minimal model and the clamp techniques are concordant. Diabetes Metab Res Rev 2010;26: 569-78.

11. Morettini M, Di Nardo F, Burattini L, Fioretti S, Gobl C, Kautzky-Willer A, Pacini G, Tura A. Assessment of glucose effectiveness from short IVGTT in individuals with different degrees of glucose tolerance. Acta Diabetol 2018;55:1011-8.

12. Marmarelis VZ, Shin DC, Zhang Y, Kautzky-Willer A, Pacini G, D’Argenio DZ. Analysis of intravenous glucose tolerance test data using parametric and nonparametric modeling: application to a population at risk for diabetes. J Diabetes Sci Technol 2013;7:952-62.

13. Tura A, Sbrignadello S, Succurro E, Groop L, Sesti G, Pacini G. An empirical index of insulin sensitivity from short IVGTT: validation against the minimal model and glucose clamp indices in patients with different clinical characteristics. Diabetologia 2010;53:144-52.

14. Pacini G, Tonolo G, Sambataro M, Maioli M, Ciccarese M, Brocco E, Avogaro A, Nosadini R. Insulin sensitivity and glucose effectiveness: minimal model analysis of regular and insulin-modified FSIGT. Am J Physiol 1998;274:E592-9.

15. Ludvik B, Waldhausl W, Prager R, Kautzky-Willer A, Pacini G. Mode of action of ipomoea batatas (Caiapo) in type 2 diabetic patients. Metabolism 2003;52:875-80.

16. Avogaro A, Watanabe RM, Dall'Arche A, De Kreutzenberg SV, Tiengo A, Pacini G. Acute alcohol consumption improves insulin action without affecting insulin secretion in type 2 diabetic subjects. Diabetes Care 2004;27:1369-74.

17. McQuaid S, O'Gorman DJ, Yousif O, Yeow TP, Rahman Y, Gasparro D, Pacini G, Nolan JJ. Early-onset insulin-resistant diabetes in obese Caucasians has features of typical type 2 diabe- 
tes, but 3 decades earlier. Diabetes Care 2005;28:1216-8.

18. Basili S, Pacini G, Guagnano MT, Manigrasso MR, Santilli F, Pettinella C, Ciabattoni G, Patrono C, Davi G. Insulin resistance as a determinant of platelet activation in obese women. J Am Coll Cardiol 2006;48:2531-8.

19. O’Gorman DJ, Yousif O, Dixon G, McQuaid S, Murphy E, Rahman Y, Gasparro D, Pacini G, Newsholme P, Nolan JJ. In vivo and in vitro studies of GAD-antibody positive subjects with type 2 diabetes: a distinct sub-phenotype. Diabetes Res Clin Pract 2008;80:365-70.

20. Tura A, Grassi A, Winhofer Y, Guolo A, Pacini G, Mari A, Kautzky-Willer A. Progression to type 2 diabetes in women with former gestational diabetes: time trajectories of metabolic parameters. PLoS One 2012; 7:e50419.

21. Stumvoll M, Mitrakou A, Pimenta W, Jenssen T, Yki-Jarvinen H, Van Haeften T, Renn W, Gerich J. Use of the oral glucose tolerance test to assess insulin release and insulin sensitivity. Diabetes Care 2000;23:295-301.

22. Diaconis P, Efron B. Computer-intensive methods in statistics. Sci Am 1983;248:116-32.

23. Tura A, Chemello G, Szendroedi J, Gobl C, Færch K, Vrbikova J, Pacini G, Ferrannini E, Roden M. Prediction of clamp-derived insulin sensitivity from the oral glucose insulin sensitivity index. Diabetologia 2018;61:1135-41.

24. Yang YJ, Youn JH, Bergman RN. Modified protocols improve insulin sensitivity estimation using the minimal model. Am J Physiol 1987;253:E595-602.

25. Finegood DT, Hramiak IM, Dupre J. A modified protocol for estimation of insulin sensitivity with the minimal model of glucose kinetics in patients with insulin-dependent diabetes. J Clin Endocrinol Metab 1990;70:1538-49.

26. Welch S, Gebhart SS, Bergman RN, Phillips LS. Minimal model analysis of intravenous glucose tolerance test-derived insulin sensitivity in diabetic subjects. J Clin Endocrinol Metab 1990; 71:1508-18.

27. Lorenzo C, Wagenknecht LE, Rewers MJ, Karter AJ, Bergman RN, Hanley AJ, Haffner SM. Disposition index, glucose effectiveness, and conversion to type 2 diabetes: the Insulin Resistance Atherosclerosis Study (IRAS). Diabetes Care 2010;33: 2098-103.

28. Chen YL, Lee SF, Pei C, Pei D, Lee CH, He CT, Liang YJ, Lin JD. Predicting glucose effectiveness in Chinese participants using routine measurements. Metab Syndr Relat Disord 2016;14: 386-90.

29. Thomaseth K, Brehm A, Pavan A, Pacini G, Roden M. Modeling glucose and free fatty acid kinetics during insulin-modified intravenous glucose tolerance test in healthy humans: role of counterregulatory response. Am J Physiol Regul Integr Comp Physiol 2014;307:R321-31.

30. Prigeon RL, Roder ME, Porte D Jr, Kahn SE. The effect of insulin dose on the measurement of insulin sensitivity by the minimal model technique. Evidence for saturable insulin transport in humans. J Clin Invest 1996;97:501-7.

31. Rayanagoudar G, Hashi AA, Zamora J, Khan KS, Hitman GA, Thangaratinam S. Quantification of the type 2 diabetes risk in women with gestational diabetes: a systematic review and meta-analysis of 95,750 women. Diabetologia 2016;59:1403-11.

32. Svensson H, Wetterling L, Andersson-Hall U, Jennische E, Eden S, Holmang A, Lonn M. Adipose tissue and body composition in women six years after gestational diabetes: factors associated with development of type 2 diabetes. Adipocyte 2018;7:229-37.

33. Hotamisligil GS, Bernlohr DA. Metabolic functions of FABPs: mechanisms and therapeutic implications. Nat Rev Endocrinol 2015;11:592-605. 